\section{LA INVESTIGACIÓN SOBRE LA DIVULGACIÓN DE LA CIENCIA EN ESPAÑA: SITUACIÓN ACTUAL Y RETOS PARA EL FUTURO}

\author{
Gregorio González-Alcaide \\ Instituto de Historia de la Medicina y de la Ciencia López Piñero, \\ Universitat de València-CSIC, Valencia, España \\ Palacio de Cerveró. Plaza Cisneros, 4. 46020 Valencia (España) \\ gregorio.gonzalez@uv.es \\ Juan Carlos Valderrama-Zurián \\ Departamento de Historia de la Ciencia y Documentación, \\ Universitat de València, Valencia, España \\ Facultad de Medicina y Odontología, \\ avenida Blasco Ibáñez, 15. 46020 Valencia (España) \\ juan.valderrama@uv.es \\ Rafael Aleixandre-Benavent \\ Universitat de València-CSIC, Valencia, España \\ Palacio de Cerveró. Plaza Cisneros, 4. 46020 Valencia (España) \\ rafael.aleixandre@uv.es
}

\section{SPANISH SCIENTIFIC RESEARCH ABOUT POPULAR SCIENCE: ACTUAL POSITION AND FUTUR CHALLENGES}

\begin{abstract}
Spanish Scientific Research about Popular Science is analyzed to identify the main scientific agents, dissemination means and subjects in this field. It is an heterogeneous area related to several scientific disciplines and purposes, stand out above them Information and Education. 286 journal articles and 50 PhD Thesis have been analyzed, observing a high increase of publications in 90s remaining until now. The five most important research cores identified are the followings: Science Journalism, Science Literacy, Linguistics and the researches related to Museums and Scientific Disciplines such as Medicine, Environment or Archaeology.
\end{abstract}

KEY WORDS: Popular Science, bibliometrics, research areas.

\section{INTRODUCCIÓN}

La Divulgación Científica puede ser definida como toda actividad encaminada a difundir el conocimiento científico y tecnológico de forma que los contenidos sean asequibles e inteligibles por una población no especializada (Belenguer Jané, 2003). Esta definición aparentemente sencilla, abar-
RESUMEN: Se analiza la producción científica española sobre divulgación de la Ciencia como objeto de investigación, identificando los principales agentes científicos, canales de difusión y ámbitos abordados. Se trata de un campo enormemente heterogéneo en el que confluyen diversas disciplinas científicas y propósitos, sobresaliendo los de informar y educar. Se han analizado 286 artículos de revista y 50 tesis doctorales. A partir de la década de los noventa es cuando se produce la eclosión de la investigación, multiplicándose el número de trabajos hasta la actualidad. Se han identificado cinco destacados núcleos temáticos de investigación: periodismo científico, alfabetización científica, lingüística, la divulgación como actividad cultural en el ámbito museístico y la investigación relacionada con determinadas disciplinas, como la Medicina, el Medio Ambiente o la Arqueología.

PALABRAS CLAVE: Divulgación de la ciencia, análisis bibliométrico, ámbitos de investigación.

ca una multiplicidad de prácticas realizadas a través de diferentes canales y en la que intervienen diversos agentes divulgadores, entre los que destacan los propios científicos, docentes, periodistas y gestores culturales.

Son numerosas las razones que justifican la necesidad y el interés por la divulgación científica, entre ellas, se puede 
destacar que resulta fundamental por la enorme complejidad y especialización de la Ciencia moderna, lo que la hace necesaria para que los propios científicos conozcan los avances en otros campos del saber; por el gran influjo que la Ciencia y la tecnología tiene en nuestras vidas cotidianas, siendo necesaria para estar al día de los últimos avances; porque la investigación es financiada en gran parte por presupuestos públicos, lo que exige una política de transparencia para que la sociedad conozca qué se está investigando y porque constituye una parte esencial de la cultura, por lo que los conocimientos científicos deben ser accesibles y formar parte del acerbo cultural básico de cualquier persona (Burkett, 1973; Junyent, 2003).

Ya desde la antigüedad clásica y a lo largo de época medieval existen numerosos ejemplos de eruditos y divulgadores que realizaron compendios de los conocimientos de su época, redactados de forma sencilla y amena para facilitar su comprensión. No será, sin embargo, hasta el desarrollo de la Revolución Científica a lo largo de los s. XVI y XVII, y sobre todo con el movimiento de la llustración en el $\mathrm{s}$. $\mathrm{XVIII}$, cuando se comience a concebir la idea de extender el conocimiento a la sociedad. A ello contribuyó la iniciativa de destacados pioneros de la divulgación, como Paracelso o Fontenelle, los gabinetes de curiosidades y la publicación de grandes compendios del conocimiento de la época que alcanzaron gran popularidad, como la Encyclopédie francesa o la Enciclopaedia Britannica, además de otras muchas publicaciones de carácter divulgativo. Ya en el $\mathrm{s}$. XIX, la divulgación científica encuentra en el desarrollo de la prensa y del periodismo un canal idóneo para llegar a capas cada vez más amplias de la población, multiplicándose la edición de periódicos, revistas y obras de carácter divulgativo, además de otras iniciativas, como la celebración de conferencias, ferias o exposiciones donde se daban a conocer los últimos avances científicos y tecnológicos. No obstante, persistirá hasta bien entrado el s. XX la división de la sociedad entre una élite de sabios o ilustrados permeables a las prácticas divulgativas de la Ciencia frente a una gran masa de población ajena a estas actividades. No será hasta finalizada la Segunda Guerra Mundial, con la generalización en la sociedad de los avances tecnológicos, la extensión de la educación y con el desarrollo de movimientos como el Public Understanding of Science en Europa y el Scientific Literacy en Estados Unidos, cuando la divulgación científica tenga una proyección mayor en la sociedad, asumiendo los científicos cada vez más la importancia de dar a conocer al gran público los nuevos descubrimientos y teorías científicas, centradas en la vida cotidiana, los problemas y necesidades de la gente, temas que han ido teniendo una presencia creciente en los medios de comunicación y que han generado el desarrollo de un periodismo especializado en temas relacionados con la divulgación científica (Raichvarg y Jacques, 1991; Fayard, 1999; Gregory y Miller, 2000; Junyent, 2003).

En relación con la importancia cobrada por la divulgación de la Ciencia, se ha desarrollado en las últimas décadas la investigación teórica sobre la misma, integrándose en el ámbito académico, siendo un tema habitual para la celebración de foros, reuniones y congresos que han favorecido la reflexión, la difusión y el intercambio de experiencias y constituyendo asimismo uno de los ámbitos prioritarios objeto de atención por parte de los gestores de las politicas científicas. El objetivo del presente trabajo consiste en analizar la producción científica española relacionada con la divulgación de la Ciencia como objeto teórico de investigación, con el propósito de caracterizar el estado de desarrollo de la investigación en el área.

\section{Metodología}

Se han identificado los artículos sobre divulgación de la Ciencia recogidos en la base de datos ISOC/Índice de Ciencias Sociales y Humanidades elaborada por el Centro de Información y Documentación Científica (CINDOC) del Consejo Superior de Investigaciones Científicas y las tesis doctorales indexadas en la base datos TESEO del Ministerio de Educación y Ciencia, mediante la ejecución de sendos perfiles de búsqueda que incluian los principales descriptores o términos que habían podido ser utilizados para la indización de los documentos sobre esta temática, como divulgación científica, comunicación científica, comunicación social y difusión de la Ciencia, así como otros términos estrechamente relacionados con la divulgación de la Ciencia, como periodismo científico, alfabetización científica y educación científica y otros más genéricos que se ha observado que eran utilizados en ocasiones para la indización de documentos relevantes una vez examinados los registros bibliográficos de los documentos recuperados, como Sociología de la Ciencia. A continuación, se ha construido una base de datos relacional con la información 
bibliográfica de los registros recuperados, procediendo al examen de todas las referencias bibliográficas con el propósito de eliminar aquellas no relevantes por no ajustarse a la materia objeto de estudio, tras examinar los títulos, descriptores y resúmenes.

Se han caracterizado mediante un análisis bibliométrico los siguientes aspectos: número de trabajos y evolución diacrónica por años de publicación, productividad de autores, instituciones y revistas fuente de publicación de los trabajos. Asimismo, se han identificado los principales ámbitos temáticos de investigación en el área y sus relaciones conceptuales, construyendo una red de relaciones terminológicas a partir de la cuantificación de la frecuencia de aparición conjunta de los descriptores asignados a los documentos. Para la realización de estos procesos se han utilizado los programas Microsoft Access y Pajek.

\section{Resultados}

Se han recuperado 286 artículos de revista sobre divulgación de la Ciencia recogidos en la base de datos ISOC publicados entre 1981 y 2005 en 94 revistas diferentes. La evolución diacrónica del número de trabajos por quinquenio (tabla 1) muestra que es a partir de la década de los noventa cuando se produce un notable incremento en el número de trabajos publicados, que se mantiene hasta la actualidad.

TABLA 1: EVOLUCIÓN DIACRÓNICA DE LOS TRABAJOS SOBRE DIVULGACIÓN CIENTÍFICA INDEXADOS EN LAS BASES DE DATOS ISOC Y TESEO (1981-2005)

\begin{tabular}{|c|c|c|}
\hline Período & $\mathrm{N}^{\circ}$ de artículos en ISOC & $\mathrm{N}^{\circ}$ de tesis en TESEO \\
\hline 1981-85 & 8 & 2 \\
\hline $1986-90$ & 23 & 3 \\
\hline 1991-95 & 50 & 12 \\
\hline $1996-00$ & 73 & 17 \\
\hline 2001-05 & 132 & 16 \\
\hline TOTAL & 286 & 50 \\
\hline
\end{tabular}

Aunque entre los principales teóricos de la divulgación científica cabe destacar autores como Manuel Calvo Hernando, Carlos Elías Pérez Casado, Pierre Fayard, Daniel E.
Jones, Leoncio López-Ocón Cabrera, Alberto Elena Díaz, Berta Marco Stiefel, Carolina Moreno Castro y Amparo Vilches Peña, todos ellos profesionales o investigadores relacionados con el periodismo científico, la Educación o la Historia de la Ciencia que han hecho de la divulgación científica una de sus principales líneas de investigación y de trabajo, hay que destacar que la divulgación de la Ciencia es un tema que ha atraido y ha sido objeto de investigación y reflexión teórica por parte de numerosos investigadores, ya que se han identificado 292 autores diferentes de muy diversas áreas de conocimiento que han participado en al menos un trabajo sobre el tema.

Asimismo, se han identificado 99 instituciones, siendo las más productivas el Consejo Superior de Investigaciones Científicas, con 15 trabajos, la Universitat de València, con 14, y la Universitat Autònoma de Barcelona y la Universidad Complutense de Madrid, con 11 trabajos cada una de ellas, respectivamente. A continuación, se sitúan la Universidad Carlos III y la Universidad del País Vasco, con nueve trabajos, la Asociación Española de Periodismo Científico, con ocho, y la Universidad de Sevilla, con siete. Otras 26 instituciones reúnen entre dos y seis trabajos y 65 las restantes un único trabajo.

Las universidades son las que encabezan el ranking de productividad en cuanto a la tipología institucional, con 156 trabajos $(68,12 \%)$. A continuación se sitúan los centros de investigación, con 22 trabajos $(9,61 \%)$ y las instituciones sin ánimo de lucro, como las asociaciones científicas, con 20 trabajos $(8,73 \%)$. Ya con un número mucho menor de trabajos se sitúan otros centros, como los museos, con ocho trabajos (3,49\%), los medios de comunicación, los centros educativos no universitarios y las empresas privadas, con seis trabajos cada uno de ellos $(2,62 \%)$, respectivamente, y finalmente, los organismos gubernamentales (gobiernos autonómicos y ayuntamientos), con cinco trabajos $(2,18 \%)$.

En cuanto a los ámbitos objeto de estudio de los artículos recogidos en ISOC, se han identificado cinco núcleos destacados de investigación relacionados con la divulgación científica, cuyas relaciones conceptuales aparecen recogidas en la figura 1. El principal de ellos, en razón del número de descriptores y la densidad de relaciones establecidas es el del periodismo científico, en el que se abordan aspectos como el papel de mediadores que ejercen los periodistas

ARBOR CLXXXV 738 julio-agosto [2009] 861-869 ISSN: 0210-1963 
entre los científicos y la sociedad, su influencia social y capacidad para generar opinión, el tratamiento que se da a la información científica, la formación profesional o la necesidad de especialización de los profesionales dedicados al mismo. Asimismo, en relación con los periodistas y el periodismo científico aparecen toda una serie de canales y medios de comunicación: la televisión, la prensa, la radio, el cine, los documentales e Internet. Un segundo ámbito de investigación destacado es el de la alfabetización científica, en el que se abordan aspectos como la educación científica y tecnológica y la divulgación y enseñanza de la Ciencia en los distintos niveles educativos. Otros dos núcleos de investigación que se han identificado están relacionados con la divulgación de la Ciencia en los museos, exposiciones y como actividad de difusión cultural y el lenguaje científico, en relación con los problemas derivados de su traducción y transposición a un lenguaje no especializado. Finalmente, hay que destacar que la divulgación de la Ciencia se relaciona con determinadas disciplinas, entre las que cabe destacar la Medicina, el Medio Ambiente y la
Arqueología, si bien, a excepción de esta última, ninguna presenta un desarrollo terminológico específico.

Se han identificado 50 tesis sobre divulgación de la Ciencia en la base de datos TESEO, ofreciendo el análisis de la evolución diacrónica de la fecha de su lectura unos resultados similares a los artículos de revista (tabla 1), ya que es a partir de la década de los noventa cuando experimenta un incremento considerable la lectura de tesis relacionadas con algún aspecto de la divulgación científica, con una media de 2,25 tesis anuales desde esa fecha. En cuanto al tema de las mismas, el periodismo científico se sitúa a la cabeza, con 29 tesis (58\%), casi todas ellas centradas en el tratamiento de las noticias científicas en los medios de comunicación, fundamentalmente sobre salud, medio ambiente o temas agroalimentarios. Destacan en cuanto al número de tesis, las leídas en las Facultades de Periodismo de la Universidad Complutense de Madrid (16), la Universitat Autònoma de Barcelona (4), la Universidad de Navarra (4) y la Universidad de La Laguna (4). Un segun-

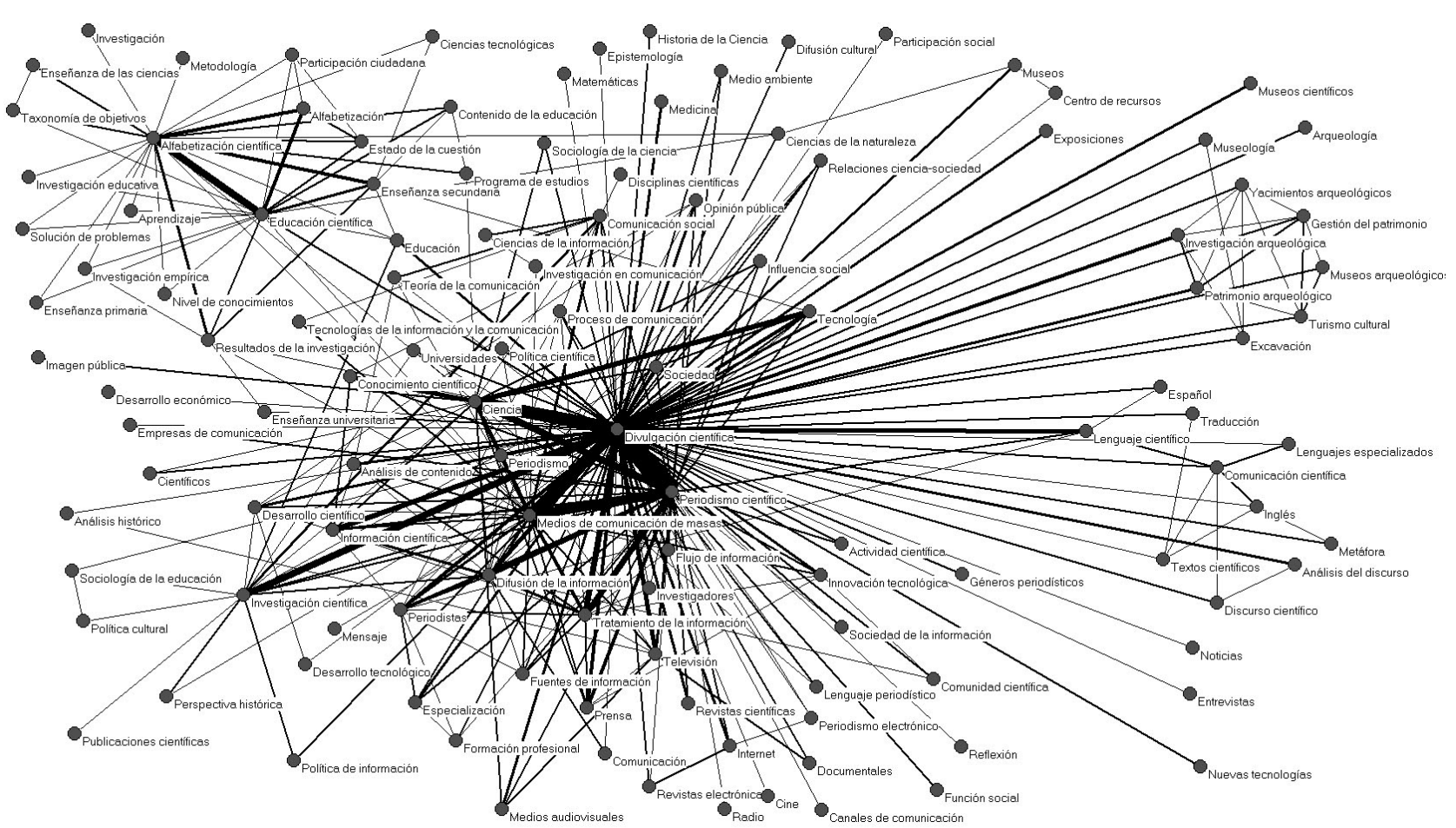

Figura 1. Red de relaciones terminológicas en los trabajos sobre divulgación científica indexados en la base de datos ISOC (> 3 trabajos). La mayor intensidad de las relaciones entre descriptores queda reflejada mediante el diferente grosor en los enlaces que vinculan cada uno de los descriptores. 
do grupo temático de tesis son los trabajos relacionados con los aspectos lingüísticos del discurso científico y su conversión al divulgativo, con seis tesis (12\%), cinco tesis que abordan la divulgación científica a través de Internet $(10 \%)$, otras cuatro tesis la alfabetización científica y museología (8\%), respectivamente, y finalmente, otras dos tesis están centradas en el análisis de aspectos históricos de la divulgación de la Ciencia (4\%).

En relación con otra destacada tipología documental donde se suelen difundir los trabajos de investigación, las comunicaciones a congresos, se puede resaltar que los cuatro congresos de Comunicación Social de la Ciencia celebrados hasta la fecha, en cuya organización han intervenido destacadas instituciones relacionadas con la divulgación de la Ciencia en España, como el Parque de las Ciencias y la Universidad de Granada (Granada, 1999), el Museo de las Ciencias Príncipe Felipe de Valencia (Valencia, 2001), Museos Científicos Coruñeses (A Coruña, 2005) y el Consejo Superior de Investigaciones Científicas (Madrid, 2007), han cumplido un destacado papel de difusión de experiencias prácticas y de reflexión teórica sobre la divulgación científica, ya que han recogido 683 trabajos, entre presentaciones, comunicaciones orales y pósteres, si bien son numerosos los foros y reuniones científicas organizados, con carácter general o monográfico, en torno a algún aspecto de la divulgación de la Ciencia, como el periodismo científico o la enseñanza y divulgación de la Ciencia (Avogadro, 2005; Izquierdo y Perales, 2006).

\section{Discusión}

Entre las limitaciones del estudio realizado se pueden apuntar las siguientes: a) La utilización de un perfil de búsqueda puede conllevar la existencia de "silencio documental", es decir, que no se recuperen todos los documentos relevantes en relación la temática objeto de estudio. Para minimizar los efectos del mismo se han tratado de incluir todas las posibles variantes utilizadas en los títulos de los trabajos y a la hora de indexar los documentos en las bases de datos, examinando los términos y descriptores incluidos en los documentos que se iban recuperando para incorporarlos al perfil de búsqueda. b) Únicamente se han estudiado dos bases de datos, y aunque se trata de dos de las principales fuentes que recogen los trabajos de investigación original en España (artículos de revista y tesis doctorales), han quedado fuera del análisis otras muchas revistas con trabajos relevantes sobre el tema objeto de estudio. c) Se ha observado que existe una gran dispersión de literatura sobre divulgación de la Ciencia, fundamentalmente en reuniones científicas de índole diversa (conferencias, foros o congresos), tratándose también de un tipo de literatura de gran importancia que requeririan de un análisis específico.

Pese a que en diversos estudios se han apuntado algunos aspectos de gran interés, la historia sobre el desarrollo de la divulgación de la Ciencia en España está aún por realizar. Dejando de lado la práctica de la divulgación por iniciativa personal de los propios científicos o divulgadores y las aportaciones foráneas, la eclosión de la reflexión teórica sobre la misma en España se sitúa a partir de mediados de la década de lo noventa. Se trata de un desarrollo tardío en relación con otros países y ámbitos geográficos, en los que se remonta al menos a la década de los setenta (Raichvarg y Jacques, 1991; Gregory y Miller, 2000) y al que han contribuido factores de índole diversa, como la consolidación de la democracia, la extensión de la enseñanza universitaria a amplias capas de la población o la creciente demanda de actividades didácticas y culturales por parte de la sociedad. Asimismo, ha tenido una importancia capital en este proceso el desarrollo y consolidación del periodismo científico especializado y el impulso propiciado, ya desde hace algunas décadas, por algunos científicos pioneros, divulgadores e instituciones que han apostado decididamente por la divulgación de la Ciencia como una parte fundamental de su trabajo y actividades.

Al igual que ocurre con gran parte de las áreas del conocimiento, son las universidades y los centros de investigación del Consejo Superior de Investigaciones Científicas los principales impulsores de la investigación teórica sobre la divulgación de la Ciencia (Chinchilla Rodríguez, 2005). Sería deseable en este sentido, el desarrollo de las condiciones necesarias para el fomento de la investigación por parte de los organismos y agentes directamente relacionados con la divulgación de la Ciencia, como los museos científicos y el desarrollo de investigaciones en colaboración entre los diferentes tipos de instituciones mencionados, con el fin de evitar la disociación entre el desarrollo teórico y la práctica divulgativa. Resulta también fundamental potenciar la existencia de canales estables de difusión de los trabajos

ARBOR CLXXXV 738 julio-agosto [2009] 861-869 ISSN: 0210-1963 
sobre divulgación de la Ciencia entre la comunidad científica, un aspecto de gran importancia en la consolidación de una disciplina o área de conocimiento junto a otros como la institucionalización académica o la existencia de un corpus de conocimiento conformado por una serie de temas objeto de investigación abordados por una comunidad de investigadores que se organizan en torno a los mismos (Bourdieu, 2003), ya que se ha observado que una parte importante de las publicaciones periódicas en las que se han difundido los trabajos han dejado de publicarse o presentan un carácter irregular en cuanto a su frecuencia de aparición y que los trabajos difundidos en congresos y otras reuniones científicas resultan en ocasiones de difícil acceso o presentan una escasa difusión y visibilidad.

Entre los ámbitos de investigación que se relacionan con la divulgación de la Ciencia, el hecho de que el periodismo científico ocupe un lugar preeminente, se justifica porque los medios informativos han ejercido desde su surgimiento, en mayor o menor medida, el papel de intermediarios entre la Ciencia y el gran público, considerando la Ciencia como noticia o como objeto de divulgación. Pese a que Josep María Casasús fija a partir de 1975 el surgimiento de lo que denomina "periodismo de servicio, del bienestar o social" (Casasús, 1988), que es aquel que se preocupa por aspectos como la calidad de vida, los temas de salud, bienestar social, el medio ambiente o por la profundización en las ventajas y prestaciones de los progresos tecnológicos, entre otros muchos aspectos estrechamente vinculados con lo que ha definido como periodismo científico, Manuel Calvo Hernando llama la atención, aún a mediados de la década de los noventa, acerca de la escasez de estudios sobre el tratamiento que recibía la Ciencia por parte de los periodistas en los medios de comunicación (Calvo Hernando, 1996). Esta situación se está viendo paliada progresivamente por la eclosión de la investigación en las dos últimas décadas puesta de manifiesto en el presente estudio, centrada en gran medida en el periodismo científico, ámbito que está recibiendo un destacado impulso a través de la celebración de congresos y seminarios específicos (Avogadro, 2005), la atención que se está prestando al mismo desde el ámbito académico con su incorporación a la formación reglada o como materia de especialización a través de formación específica de posgrado y como objeto de investigación en programas de doctorado (Pastor Ruiz, 2002; Aranes Usandizaga y Landa Montenegro, 2002).
En relación con el periodismo cientifico, destacan tres elementos: los agentes implicados, las fuentes de información y los distintos canales o medios utilizados. El interés por los agentes implicados responde a que uno de los principales debates que se suscita a nivel teórico y en los foros sobre divulgación de la Ciencia que reúnen a periodistas y científicos, es el papel que debe asumir el periodismo científico. En relación con ello, existen básicamente dos concepciones contrapuestas: la de aquellos que defienden que el periodismo científico se debe limitar a informar sobre la actualidad científica y la de los que postulan que los periodistas científicos deben asumir una función "formativa" (Belenguer Jané, 2003). En este sentido, hay que señalar que la divulgación científica es una tarea compleja y delicada, que exige del dominio de múltiples destrezas y competencias. Aldo Borsese apunta que es fundamental que el divulgador posea una formación específica que profundice en tres niveles: los contenidos científicos, el lingüistico y el psicopedagógico (Borsese, 1999). Aunque estos tres niveles están presentes en el diagrama terminológico de la investigación sobre la divulgación de la Ciencia que se ha confeccionado, cabe resaltar que no están interrelacionados entre sí, sino que aparecen de forma aislada. En cuanto a las fuentes de información utilizadas en el ámbito periodístico encontramos en el diagrama las revistas científicas. En relación con ellas, algunos autores han llamado la atención sobre el hecho de que se utilizan sólo un número muy reducido de las mismas, fundamentalmente Nature y Science, lo que se explica por la credibilidad que se atribuye a estas revistas y porque poseen importantes gabinetes de prensa que redactan los contenidos en lenguaje periodístico, si bien esta limitación puede conllevar numerosos problemas, como la lejanía de las fuentes originales o que se primen las investigaciones en función de su noticiabilidad (Elías, 2002a). Asimismo, algunos estudios han alertado sobre el hecho de que las fuentes de información periodística en ocasiones no son los científicos, sino otros agentes como empresas, administraciones u ONG (Montaño Montaño, 1999). Finalmente, en lo referente a los canales de difusión, destacan fundamentalmente la televisión y la prensa, lo que se justifica por tratarse de dos de los principales medios de comunicación de masas, así como Internet, lo que hay que poner en relación con el reciente desarrollo experimentado por este medio y las posibilidades que ofrece para la divulgación científica (Meso Ayerdi y Díaz Noci, 2002). 
El segundo de los temas destacados en relación con la investigación sobre la divulgación de la Ciencia es la alfabetización científica, que puede ser definida como el conocimiento práctico y cotidiano de la Ciencia, de forma que se comprenda la naturaleza global de la misma, del proceso científico y de las relaciones entre Ciencia, tecnología y sociedad (Valentín, 2005). Los aspectos recogidos en el diagrama conceptual reflejan los planteamientos de diversos investigadores que postulan que se debe alcanzar un nivel de comprensión científico básico en la sociedad, lo que pasa por la implantación de una cultura científica y la integración de la didáctica de las ciencias en el ámbito educativo (Escalas, 1999). En este sentido, se debe profundizar en el desarrollo de investigaciones teóricas en relación con la alfabetización científica en las que educadores, científicos, sociólogos, lingüistas y otros profesionales fijen los aspectos pedagógicos, contenidos, conceptos y vocabulario que la deben integrar en los diferentes niveles educativos (Escalas, 1999; Gavidia, 2005), si bien la alfabetización científica es un aspecto que debe trascender el ámbito educativo para extenderse a toda la vida de las personas y a toda la sociedad (Goirena de Gandarias y Garea Lafuente, 2002).

Otro de los temas centrales de investigación teórica responde a las dificultades lingüisticas que plantea la información científica a la hora de ser transmitida a un público no especializado, ya que la divulgación de la Ciencia tiene que hacer frente a la dificultad que supone hacer comprensible un discurso especializado que se caracteriza por un complejo y elaborado sistema conceptual. Para ello, es necesario reelaborar este discurso, lo que ha generado numerosos estudios que desde el ámbito de la lingüística han analizado las características del discurso científico frente al divulgativo, proponiendo modelos y pautas para su conversión o reelaboración (Ciapuscio, 2000; Galán Rodríguez, 2003; Alcíbar, 2004).

En cuanto a las disciplinas objeto de investigación en relación con la divulgación científica, el hecho de que en el diagrama terminológico solamente están presentes un número muy reducido de las mismas, puede responder a que sean ámbitos más fácilmente "popularizables" o que se trate de disciplinas de gran relevancia o interés para el público (Calvo Hernando, 1996). Destaca en este sentido la Medicina, cuyo interés en relación con la divulgación científica se justifica porque aborda temas que afectan directamente a la vida de las personas y que por tanto resultan de interés para los lectores de cualquier medio de comunicación, explicación que es válida también en relación con otras áreas como el Medio Ambiente (Aranes Usandizaga y Landa Montenegro, 2002; Goirena de Gandarias y Garea Lafuente, 2002). Cabe destacar asimismo la importancia de la Arqueología, lo que se explica en este caso porque la divulgación constituye uno de los pilares de la disciplina, recalcándose siempre a nivel teórico en la misma, la importancia de dar a conocer los conocimientos a la sociedad como el último estadio en el desarrollo de cualquier investigación arqueológica (Querol y Martínez, 1996). Llama la atención en cambio la ausencia de otras muchas disciplinas, probablemente reflejo de la ausencia de una investigación teórica sobre la divulgación científica en las mismas. Frente a ello, se debería fomentar entre los científicos una cultura de la divulgación, para que se ejerza la misma como una parte fundamental más de su trabajo, ya que aunque cada vez son más frecuentes los ejemplos de científicos que ejercen el papel de divulgadores de sus propios trabajos e investigaciones (Calvo Hernando, 1996), todavía la divulgación ejercida por los propios científicos tiene un escaso peso, lo que se puede explicar por el hecho de que la divulgación científica no siempre ha sido considerada positivamente. En este sentido, hasta hace pocas fechas, muchos científicos veían con desdén las actividades de los colegas que se dedicaban a la divulgación del conocimiento y, aún hoy, se considera habitualmente la divulgación como una actividad secundaria o menor, cuando no totalmente innecesaria y que puede incluso llegar a perjudicar la carrera académica (Junyent, 2003). Para hacer frente a esta situación resulta fundamental que los gestores de las políticas científicas y los sistemas de evaluación y reconocimientos de las carreras académicas e investigadoras favorezcan que los científicos realicen tareas de divulgación o comunicación social de la Ciencia.

Las principales conclusiones del estudio realizado son las siguientes: a) A pesar de su desarrollo tardío en relación con otros ámbitos geográficos, las investigaciones y reflexiones teóricas acerca de la divulgación de la Ciencia en España han experimentado un considerable crecimiento en los últimos años, reflejo de la importancia e interés concedido a la misma. b) La investigación es impulsada fundamentalmente desde el ámbito universitario y por parte de los centros de investigación. c) La mayor parte de las investigaciones teóricas sobre divulgación de la 
Ciencia están relacionadas con el periodismo científico y los medios de comunicación y en menor medida con la alfabetización científica y la Lingüistica, destacando entre las disciplinas científicas la Medicina, el Medio Ambiente y la Arqueología; y d) llama la atención el escaso peso de los científicos y de los museos, en tanto que los principales agentes vinculados con la actividad científica y los principales ámbitos responsables de hacer llegar la Ciencia a la sociedad en un contexto de enseñanza no reglada, respectivamente, así como el reducido número de disciplinas vinculadas con la divulgación de la Ciencia que se han identificado.

En relación con estas conclusiones y con todos los aspectos mencionados a lo largo del trabajo, se pueden esbozar algunos de los retos futuros que se deberian acometer en relación con la divulgación de la Ciencia, que pasan por una mayor implicación en el desarrollo teórico sobre la misma de todas las disciplinas y áreas del conocimiento, rompiendo la tradicional división bipolar entre disciplinas más o menos popularizables y entre Ciencias y Humanidades (Elias, 2002b; Pérez Cobo, 2002); también de los museos de Ciencia, en tanto que espacios divulgativos por excelencia en nuestra sociedad (Aguirre Pérez y Vázquez Molini, 2004); y finalmente, de los propios científicos, que deben asumir un mayor protagonismo, que no pasa necesariamente por ejercer el papel de "divulgadores" activos, pero sí por informar y poner a disposición de los periodistas y otros colectivos sus conocimientos, teorías y descubrimientos (Calvo Hernando, 2002). Asimismo, resulta fundamental una mayor potenciación de la alfabetización científica en el ámbito educativo, ya que la actitud e interés por la Ciencia se configura en gran medida durante las etapas de formación primaria y secundaria (Blanco López, 2004). Otros aspectos sobre los que debería incidir son el análisis preciso de las características del tipo de público al que van destinadas las prácticas divulgativas y la forma en la que los mensajes informativos que se originan a través de la divulgación científica llegan a los destinatarios (Borsese, 1999). Finalmente, no se puede olvidar el papel de Internet, hoy en día el principal medio de comunicación global y un canal idóneo para la práctica de la divulgación científica por las múltiples posibilidades que ofrece, debiéndose profundizar en el estudio acerca de cómo se ejerce la divulgación en este medio por parte de las instituciones tradicionalmente vinculadas a la prácticas divulgativas, como universidades o museos, así como analizar el desarrollo de nuevas iniciativas divulgativas, como los museos digitales (Meso Ayerdi y Díaz Noci, 2002).

\section{AGRADECIMIENTOS}

Este trabajo ha sido realizado gracias a la financiación del programa I3P cofinanciado por el Fondo Social Europeo del Consejo Superior de Investigaciones Científicas.

\section{BIBLIOGRAFÍA}

Aguirre Pérez, Constancio y Vázquez Moliní, Ana María (2004): "Consideraciones generales sobre la alfabetización científica en los museos de la ciencia como espacios educativos no formales", Revista Electrónica de Enseñanza de las Ciencias, 3 (3).

Recibido: 20 de diciembre de 2007 Aceptado: 15 de febrero de 2008
Alcibar, Miguel (2004): "La divulgación mediática de la Ciencia y la tecnología como recontextualización discursiva", Anàlisi: Quaderns de Comunicació $i$ Cultura, 31, 43-70.

Aaranes Usandizaga, José Ignacio y Landa Montenegro, Carmelo (2002): "Periodismo científico: conceptualización y líneas de investigación", Mediatika: Cuadernos de Medios de Comunicación, 8, 293-319.

Avogadro, Marisa (2005): "Periodismo de la Ciencia: aproximaciones y cronología", Razón y Palabra, 43.

Blanco López, Ángel (2004): "Relaciones entre la educación científica y la divulgación de la ciencia", Revista Eureka sobre Enseñanza y Divulgación de las Ciencias, 2004, 1 (2): 70-86.

Belenguer Jané, Mariano (2003): "Información y divulgación científica: dos con- 
ceptos paralelos y complementarios en el periodismo científico", Estudios sobre Mensaje Periodístico, 9, 43-53.

Bourdieu, Pierre (2003): El oficio de científico. Ciencia de la ciencia y reflexividad, Barcelona, Anagrama.

Burkett, David Warren (1973): Writing science news for the mass media, Houston (Texas), Gulf Publishing Company.

Calvo Hernando, Manuel (1996): "La divulgación de la Ciencia como objeto de investigación", Arbor: Ciencia, pensamiento y cultura, 601, 105-120.

Calvo Hernando, Manuel (2002): "El periodismo científico, necesario en la sociedad actual", Mediatika: Cuadernos de Medios de Comunicación, 2002, 8, 485-498.

Casasús, Josep Maria (1988): Iniciación a la periodistica: manual de comunicación escrita y redacción periodística informativa, Barcelona, Teide.

Chinchilla Rodríguez, Zaida (2005): Indicadores bibliométricos de la actividad científica española, 2004, Madrid, Fundación Española para la Ciencia y la Tecnología.

Ciapuscio, Guiomar (2000): "Hacia una tipología del discurso especializado", Revista Iberoamericana de Discurso y Sociedad, 2 (2), 39-71.

Elias, Carlos (2002a): "Influencia de las revistas de impacto en el periodismo científico y en la Ciencia actual", Revista Española de Investigaciones Sociológicas, 98, 123-137.
Elias, Carlos (2002b): "Periodistas especializados en ciencia: formación, reconocimiento e influencia", Mediatika: Cuadernos de Medios de Comunicación, 8, 389-403.

Escalas, Teresa (1999): "Un análisis de la divulgación científica en Cataluña", Alambique: Didáctica de las Ciencias Experimentales, 21, 35-40.

Fayard, Pierre (1999): "La sorpresa de Copérnico: jel conocimiento gira alrededor del público!", Alambique: Didáctica de las Ciencias Experimentales, 21, 9-16.

Galán Rodríguez, Carmen (2003): "La Ciencia en zapatillas: análisis del discurso de divulgación científica", Anuario de Estudios Filológicos, 26, 137-156.

Gavidia, Valentín (2005): "Los retos de la divulgación y la enseñanza científica en el próximo futuro", Didáctica de las Ciencias Experimentales y Sociales, 19, 91-102.

Goirena de Gandarias, Juan José y Garea Lafuente, María Gloria (2002): "La salud de la información científica", Mediatika: Cuadernos de Medios de Comunicación, 8, 523-558.

Gregory, Jane y Miller, Steve (2000): Science in public: Communication, culture and credibility, Cambridge (Massachussets), Perseus Books.

Izquierdo, Mercé y Perales, Javier (2006): "Actas del VII Congreso Internacional sobre Investigación en la Didáctica de las Ciencias", Revista Eureka sobre En- señanza y Divulgación de las Ciencias, 2006, 3 (2): 312-314.

Junyent, Cristina (2003): "Comunicació científica i percepció social de la Ciencia", Coneiximent i Societat: Revista d'Universitats, Recerca i Societat de la Informació, 2, 6-15.

Meso Ayerdi, Koldobika y Díaz Noci, Javier (2002): "Periodismo científico en el ciberespacio: la información académica al encuentro de la tecnología digital", Mediatika: Cuadernos de Medios de Comunicación, 8, 605-629.

Montaño Montaño, Miguel (1999): "El Canal 2 de Andalucía y la información ambiental: el programa Espacio Protegido", Ámbitos: Revista Internacional de Comunicación, 2, 207-227.

Pastor Ruiz, Fátima (2002): "Periodismo científico y Documentación: estrategia y herramientas de búsqueda", Mediatika: Cuadernos de Medios de Comunicación, 8, 207-218.

Pérez Cobo, José Carlos (2002): "Sonrisas muy serias o la importancia del periodismo científico", Mediatika: Cuadernos de Medios de Comunicación, 8, 349-373.

Querol, María Ángeles y Martínez Díaz, Belén (1996): La gestión del patrimonio arqueológico en España, Madrid, Alianza.

Raichvarg, Daniel y Jacques, Jean (1991), Savants et ignorants: une histoire de la vulgarisation des sciences, Paris, Éditions du Seuil. 